\title{
Study on electron stochastic motions in the magnetosonic wave field: Test particle simulations
}

\author{
Kai Fan ${ }^{1,2}$, XinLiang Gao ${ }^{1,2^{*}}$, QuanMing Lu ${ }^{1,2}$, and Shui Wang ${ }^{1,2}$ \\ 'CAS Key Laboratory of Geospace Environment, Department of Geophysics and Planetary Science, University of Science and Technology of China, Hefei \\ 230026, China; \\ ${ }^{2}$ CAS Center for Excellence in Comparative Planetology, Hefei 230026, China
}

\section{Key Points:}

- Test particle simulations show that electron motion can become stochastic in the MS wave field when the wave amplitude exceeds a certain threshold.

- We have thoroughly studied the effects of bounce resonance order, pitch angle, and wave frequency on the amplitude threshold.

- The coexistence of bounce and Landau resonances induced by MS wave can significantly reduce the amplitude threshold.

Citation: Fan, K., Gao, X. L., Lu, Q. M., and Wang, S. (2021). Study on electron stochastic motions in the magnetosonic wave field: Test particle simulations. Earth Planet. Phys., 5(6), 592-600. http://doi.org/10.26464/epp2021052

\begin{abstract}
Using the test particle simulation method, we investigate the stochastic motion of electrons with energy of 300 keV in a monochromatic magnetosonic (MS) wave field. This study is motivated by the violation of the quasi-linear theory assumption, when strong MS waves (amplitude up to 1 nT) are present in the Earth's magnetosphere. First, electron motion can become stochastic when the wave amplitude exceeds a certain threshold. If an electron initially resonates with the MS wave via bounce resonance, as the bounce resonance order increases, the amplitude threshold of electron stochastic motion increases until it reaches the peak at about the 11 th order in our study, then the amplitude threshold slowly declines. Further, we find that the coexistence of bounce and Landau resonances between electrons and MS waves will significantly reduce the amplitude threshold. In some cases, the electron motion can become stochastic in the field of an MS wave with amplitudes below $1 \mathrm{nT}$. Regardless, if neither the bounce nor Landau resonance condition is satisfied initially, then the amplitude threshold of stochastic motion shows an increasing trend for lower frequencies and a decreasing trend for higher frequencies, even though the amplitude threshold is always very large (> $5 \mathrm{nT})$. Our study suggests that electron stochastic motion should also be considered when modeling electron dynamics regulated by intense MS waves in the Earth's magnetosphere.
\end{abstract}

Keywords: magnetosonic waves; electron stochastic motions; bounce resonance; test particle simulations

\section{Introduction}

Magnetosonic (MS) waves, also referred to as equatorial noise or ion Bernstein mode, are electromagnetic emissions at frequencies between the proton gyrofrequency $f_{\mathrm{cp}}$ and lower hybrid resonance frequency $f_{\mathrm{LHR}}$ in the Earth's magnetosphere (Russell et al., 1970; Gurnett, 1976; Santolík et al., 2002, 2004; Gary et al., 2011; Boardsen et al., 2016). They are usually detected at several harmonics of the local proton gyrofrequency in the time-frequency spectrogram (Balikhin et al., 2015; Boardsen et al., 2018), while intense MS waves can also have a continuous spectrum (Tsurutani et al., 2014). The discrete and continuous nature of MS waves has been well-explained by Chen LJ et al. (2016) and SUN JC et al. $(2016 a, b)$. Satellite observations indicate that MS waves are confined within a few degrees of the magnetic equator and located at $2 \leq L$-shell $\leq 8$ (Perraut et al., 1982; Laakso et al., 1990; Ma QL et al.,

Correspondence to: X. L. Gao, gaoxl@mail.ustc.edu.cn

Received 25 MAY 2020; Accepted 02 AUG 2021.

Accepted article online 15 OCT 2021.

(C) 2021 by Earth and Planetary Physics.
2013), which are commonly believed to be excited by the ring distribution of ring-current protons (Boardsen et al., 1992; Meredith et al., 2008; Chen LJ et al., 2010; Ma QL et al., 2014; Yu J et al., 2019b). MS waves typically have a very large wave normal angle (WNA) that is close to $90^{\circ}$ and are nearly linear polarized (Kasahara et al., 1994; Chen LJ and Thorne, 2012; Min K et al., 2019). The dominant magnetic component of MS waves is along the background magnetic field (i.e., large magnetic compressibility), while the wave electric field is nearly aligned with the wave vector (Boardsen et al., 2016, 2018; Gao XL et al., 2018; Sun JC et al., 2020). Statistical results show that MS waves have average amplitudes of $\sim 50$ pT in the inner magnetosphere (Ma QL et al., 2013), whereas very intense MS waves have also been reported with amplitudes up to $\sim 1$ nT during substorm activity (Tsurutani et al., 2014; Teng SC et al., 2019).

Energetic electrons trapped in Earth's Van Allen radiation belts typically experience three periodic motions: gyration, bounce, and drift. These three periodic motions are decoupled in the magnetosphere because of their well-separated periods, and are associated with the first, second, and third adiabatic invariants, re- 
spectively (Schulz and Lanzerotti, 1974). The violation of these adiabatic invariants can be caused by several plasma waves through resonances with electrons, leading to irreversible, dynamic changes of electron distribution (Thorne, 2010). In general, these resonant wave-particle interactions can be described in terms of pitch angle and energy diffusion coefficients based on quasi-linear theory, which has been widely employed to study interactions between MS waves and electrons in the magnetosphere (Horne et al., 2007; Li XX et al., 2015; Maldonado and Chen LJ, 2018; Fu S et al., 2019a). MS waves are capable of accelerating electrons between $\sim 10 \mathrm{keV}$ and $\sim 100 \mathrm{keV}$ to relativistic energies via Landau resonance, indicating they might play an important role in radiation belt dynamics (Horne et al., 2007). Further, MS wave-driven resonance interaction is believed to be a candidate mechanism responsible for the formation of electron butterfly distributions whose local flux has a minimum value at $90^{\circ}$ pitch angle in the magnetosphere (Xiao FL et al., 2015; Li JX et al., 2016; Ma QL et al., 2016; Maldonado et al., 2016; Ni BB et al., 2018; Yu J et al., 2019a).

The motion of a charged particle in a wave field can become stochastic (i.e., chaos) if the wave amplitude exceeds a certain threshold, resulting in the nonlinear diffusion of particles in pitch angle and energy (Smith and Kaufman, 1978; Lichtenberg and Lieberman, 1992; Chen L et al., 2001; Gates et al., 2001). Theoretical and simulation studies demonstrate that the stochastic motion of charged particles occurs when neighboring resonances overlap in phase space, and the width of the resonant island is mainly controlled by the wave amplitude (Villalón and Burke, 1987; Guo ZH et al., 2008; Lu QM and Chen L, 2009). A Poincare plot is the intersection of an orbit in the state space of a continuous dynamical system with a certain lower-dimensional subspace, which is a useful tool to analyze a dynamical system and approximately determine the amplitude threshold of stochastic motion. The other way to derive the stochastic threshold is Fast Fourier Transform (FFT) analysis of the particle velocity time series, in which the transition between the discrete and continuous power spectra appears. Both methods have been widely used to study ion dynamics in the Alfven/ion cyclotron or MHD MS wave field (Chen $L$ et al., 2001; White et al., 2002; Guo ZH et al., 2008; Lu QM and Chen L, 2009; Gao XL et al., 2012).

In this paper, we thoroughly study the electron stochastic motion induced by a monochromatic MS wave with the fully relativistic test particle simulation method, which may violate the assumption of the quasi-linear theory (QLT). We have given the amplitude thresholds of electron stochastic motion in the MS wave field in various cases and also investigated their dependences on wave and particle parameters. Importantly, the results reveal that the amplitude threshold of stochastic motion for $\sim 100 \mathrm{keV}$ electrons can be less than $1 \mathrm{nT}$ in some cases. This suggests that electron stochastic motion may be induced by MS waves in the Earth's magnetosphere, and therefore the validity of QLT may be questionable when intense MS waves are involved. In Section 2, the test particle model is described followed by simulation results in Section 3, concluding with a summary and discussion in Section 4.

\section{Simulation Model}

We adopt a test particle simulation model to study electron mo- tion in the field of a monochromatic MS wave. Here, the background magnetic field $\boldsymbol{B}_{0}$ is a simplified dipole field without considering the curvature, given as $\boldsymbol{B}_{0}=B_{0 x} \boldsymbol{e}_{x}+B_{0 y} \boldsymbol{e}_{y}+B_{0 z} \boldsymbol{e}_{z}$ in the Cartesian coordinate system, where $z$ is the distance along the field line from the equatorial plane. $B_{0 z}$ is given as a function of magnetic latitude $\lambda$ as $B_{0 z}(\lambda)=B_{0 z}(\lambda=0) \sqrt{1+3 \sin ^{2} \lambda} / \cos ^{6} \lambda$, and in order to satisfy $\nabla \cdot \boldsymbol{B}_{0}=0$, we simply require $B_{0 x}=-0.5 x\left(\mathrm{~d} B_{0 z} / \mathrm{dz}\right)$ and $B_{0 y}=-0.5 y\left(\mathrm{~d} B_{0 z} / \mathrm{d} z\right)$. The relation between $z$ and $\lambda$ is described by $\mathrm{d} z=L R_{\mathrm{E}} \sqrt{1+3 \sin ^{2} \lambda} \cos \lambda \mathrm{d} \lambda$ in our model, where $L$ is the $L$-shell value and $R_{\mathrm{E}}$ is the radius of Earth. In this simplified dipole field model, we have neglected the drift motion of electrons by assuming that MS waves are distributed uniformly over magnetic local time, which has also been used in previous works (Tao X et al., 2012; Fu S et al., 2019a).

In this model, the chosen simulation domain is located at the magnetic field line of $L=6.8$, meaning the trajectory of the test particle is fixed at this field line (i.e., the $z$-axis). Here the choice of a relatively larger $L$ allows that the bounce resonances between MS waves and electrons can occur for the first few orders (Shprits, 2009). Satellite observations indicate that MS waves are typically detected near the magnetic equator, so MS waves in our model are confined within $|\lambda| \leq 3^{\circ}$ and have a constant amplitude in this region. The WNA of MS waves is fixed as $89^{\circ}$, and its wave vector lies in the $x-z$ plane. To reduce the transit time effect, the ratio between plasma frequency and electron gyrofrequency $\left(\omega_{\mathrm{pe}} / \Omega_{\mathrm{e} 0}\right)$ is set relatively higher, i.e., 32 .

The electrons' motion in our simulation model can be described by the following equations:

$$
\begin{gathered}
\gamma m_{0} \frac{\mathrm{d} \boldsymbol{v}}{\mathrm{d} t}=q\left[\boldsymbol{E}_{\mathrm{w}}+\boldsymbol{v} \times\left(\boldsymbol{B}_{0}+\boldsymbol{B}_{\mathrm{w}}\right)\right], \\
\frac{\mathrm{d} z}{\mathrm{~d} t}=v_{z^{\prime}}
\end{gathered}
$$

where $\gamma$ is the Lorentz factor, and $m_{0}$ and $q$ are the rest mass and charge of the electron, respectively. The wave field $E_{\mathrm{w}}$ and $\boldsymbol{B}_{\mathrm{w}}$ at a specific position and time is given by:

$$
\begin{aligned}
& \boldsymbol{E}_{\mathrm{w}}=\boldsymbol{e}_{x} E_{x}^{w} \sin \phi+\boldsymbol{e}_{y} E_{y}^{w} \cos \phi+\boldsymbol{e}_{z} E_{z}^{w} \sin \phi, \\
& \boldsymbol{B}_{\mathrm{w}}=\boldsymbol{e}_{x} B_{x}^{w} \cos \phi+\boldsymbol{e}_{y} B_{y}^{w} \sin \phi+\boldsymbol{e}_{z} B_{z}^{w} \cos \phi,
\end{aligned}
$$

where $\phi=\int k \mathrm{~d} r-\omega t+\phi_{0}$ is the wave phase, and the wave normal vector $\boldsymbol{k}$ is given by $\boldsymbol{k}=(k \sin \theta, 0, k \cos \theta) . \omega$ is the wave frequency, and $\phi_{0}$ is the initial wave phase at the equator of $L=6.8$. Here, the wave number $k$, as well as each component of the wave amplitude, i.e., $E_{x}^{w}, E_{y}^{w}, E_{z}^{w}, B_{x}^{w}, B_{y}^{w}$, and $B_{z}^{w}$ can be determined by combining the cold plasma dispersion relation of MS waves and the Maxwell equation $i \boldsymbol{k} \times \boldsymbol{E}_{\mathrm{w}}=\mathrm{i} \omega \boldsymbol{B}_{\mathrm{w}}$ (e.g., Tao $\mathrm{X}$ et al., 2012; Tao $\mathrm{X}$ and Li X, 2016; Fu S et al., 2016, 2019b)

$$
\begin{gathered}
\frac{B_{x}^{w}}{B_{z}^{w}}=-\frac{1}{\tan \theta^{\prime}} \\
\frac{B_{y}^{w}}{B_{z}^{w}}=-\frac{P\left(S-N^{2}\right)}{D \tan \theta\left(N^{2} \sin ^{2} \theta-P\right)^{\prime}} \\
\frac{E_{x}^{w}}{B_{z}^{w}}=\frac{C\left(N^{2}-S\right)}{D N \sin \theta},
\end{gathered}
$$




$$
\begin{gathered}
\frac{E_{y}^{w}}{B_{z}^{w}}=\frac{c}{N \sin \theta^{\prime}} \\
\frac{E_{z}^{w}}{B_{z}^{w}}=\frac{c N \cos \theta\left(S-N^{2}\right)}{D\left(P-N^{2} \sin ^{2} \theta\right)}, \\
B_{\mathrm{w}}=\sqrt{\left(B_{x}^{w}\right)^{2}+\left(B_{y}^{w}\right)^{2}+\left(B_{z}^{w}\right)^{2}} .
\end{gathered}
$$

Here, $\theta$ is the WNA of the MS wave, $P, S$, and $D$ are the Stix parameters, $N=c k / \omega$ is the refractive index, and $c$ is the light speed. The Boris method (Boris, 1970) is utilized to solve the fully relativistic Lorentz Equation (1), which has been widely used in previous works (Lu QM and Chen L, 2009; Gao XL et al., 2012; Fu S et al., 2019a, b; Cai B et al., 2020; Fu S and Ge YS, 2021). Note that we only use Equation (1) to trace the charged particle motion in the analytical wave field, where wave-particle interactions are nonself-consistent. In each simulation run, we initially set the selected electron at the equator of $L=6.8$, and the initial gyro-phase angle (i.e., the angle between $v_{\perp}$ and the $x$-axis) of the electron is fixed as zero (i.e., $v_{x}=v_{\perp}$ and $v_{y}=0$ ). The time step is set as $0.1 \Omega_{\mathrm{e} 0}^{-1}$, and the total time for each simulation case is $500 \tau_{\mathrm{b} 0}$, where $\tau_{\mathrm{b} 0}$ is the unperturbed bounce period of the selected electron. The electron's velocity is normalized by $v_{\mathrm{th}}$, which is the thermal velocity of electrons with a temperature of $1 \mathrm{eV}$.

In this study, we consider both the bounce resonance and Landau resonance between electrons and MS waves. The bounce resonance condition is $\omega=n \omega_{\mathrm{b}}$, where $n$ is the bounce resonance order, and $\omega_{\mathrm{b}}=2 \pi / \tau_{\mathrm{b}}$ is the electron's bounce frequency that is estimated by $\tau_{\mathrm{b}} \approx 0.085 \mathrm{~L}(c / v)\left(1.3-0.56 \sin a_{\text {eq }}\right)$ (Lyons and Williams, 1984, p. 24). Note that, since the bounce resonance occurs over a finite range of $\omega$ near $n \omega_{\mathrm{b}}(n=1,2,3 \ldots)$, i.e., not necessarily at exact $n \omega_{\mathrm{b}}$ (Chen LJ et al., 2015), the bounce resonance condition can be well-satisfied initially. The Landau resonance condition is given by $\omega=k_{/ /} v_{/ /}$, where $k_{/ /}$is the parallel wave number and $v_{/ /}$is the parallel velocity of the electron. There exist two methods to exhibit the stochastic motion of the electron in the literature. The first is that the trajectory of the electron in the Poincare map is irregular, while the second is that the frequency spectrum of electron velocity is continuous.

\section{Simulation Results}

We first investigate electron motion in the wave field when the bounce resonance satisfies at the first order. Figure 1 displays the spectrum of the $z$ component of electron velocity $v_{z}$ as a function of frequency, which is obtained by the Fast Fourier Transform (FFT) time series of $v_{z}(t)$ from $t=0$ to $t=500 \tau_{\mathrm{b} 0}$. The parameters of the monochromatic MS wave are listed here: $\omega=\omega_{\mathrm{b} 0}$, initial phase at the equatorial plane $\phi_{0}=0.5$, and amplitude (a) $B_{\mathrm{w}}=0.1 \mathrm{nT}$, (b) $B_{\mathrm{w}}=0.7 \mathrm{nT}$, and (c) $B_{\mathrm{w}}=3.0 \mathrm{nT}$. The initial equatorial pitch angle $a_{\text {eq0 }}$ and kinetic energy $E_{k 0}$ of the selected electrons are $70^{\circ}$ and $300 \mathrm{keV}$, i.e., with the corresponding bounce period $\tau_{\mathrm{b} 0}=0.58 \mathrm{~s}$ and bounce angular frequency $\omega_{\mathrm{b} 0}=10.9 \mathrm{rad} / \mathrm{s}$. When the wave amplitude is very small $(0.1 \mathrm{nT})$, the spectrum shows several finite power peaks (Figure 1a) where each peak corresponds to one periodic bounce motion of an electron. Here, the change of energy and pitch angle of the electrons caused by MS wave interaction leads to the change of bounce period of the electrons, and the existence of finite power peaks in the spectrum means the electron motion is quasi-periodic. As the amplitude increases, the spectrum of $v_{z}$ tends to have more and more power peaks (Figure 1b) until it becomes continuous (Figure 1c). In other words, the motion of the electrons becomes stochastic in the field of MS waves with a sufficiently large amplitude. This method of presenting the stochastic motion of charged particles in a wave field was developed by Lu QM and Chen L (2009). In this case, the amplitude threshold of electron stochastic motion is estimated as $0.7 \mathrm{nT}$. It is worth mentioning that, although the threshold estimated in this study is not very accurate, the dependences of the amplitude threshold on wave and particle parameters (i.e., the main conclusions in this paper) remain unchanged.

The electron stochastic motion is also illustrated by the Poincare map in Figure 2. Figures $2 \mathrm{a}, 2 \mathrm{~d}$, and $2 \mathrm{~g}$ and Figures $2 \mathrm{~b}, 2 \mathrm{e}$, and $2 \mathrm{~h}$ give the overview and partially enlarged view, respectively, of the scatterplots of the electron's trajectory in $\left(z, v_{z}\right)$ space,. The black line in Figure $2 \mathrm{a}, 2 \mathrm{~d}$, or $2 \mathrm{~g}$ represents the Landau resonance velocity as a function of $z$. Further, Figure $2 c, 2 f$, and $2 i$ show the Poincare map obtained by recording points when the electron crosses the equator from south to north (i.e., $z=0$, and $v_{z}>0$ ); the para-
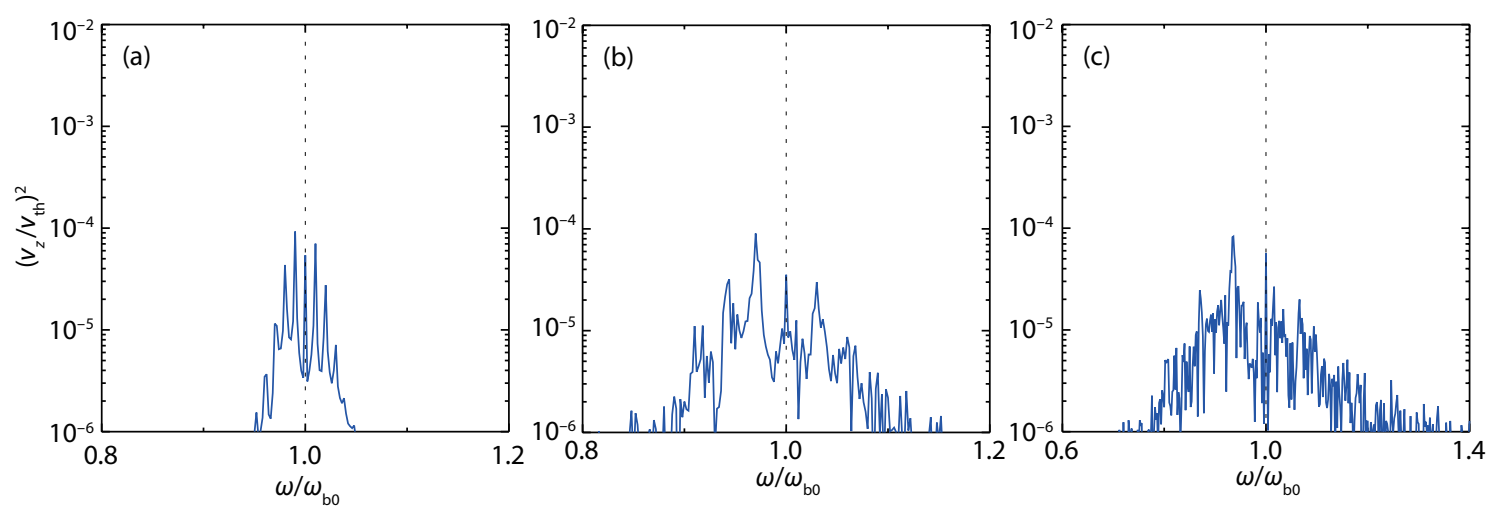

Figure 1. The spectrum of the $z$ component of electron velocity $v_{z}$ as a function of frequency, which is obtained by the Fast Fourier Transform (FFT) the time series of $v_{z}(t)$ from $t=0$ to $t=500 \tau_{\mathrm{b} 0}$. The parameters of the monochromatic MS wave are listed here: $\omega=\omega_{\mathrm{b} 0}$, initial phase at the equatorial plane $\varphi_{0}=0.5 \pi$, and amplitude (a) $B_{\mathrm{w}}=0.1 \mathrm{nT}$, (b) $B_{\mathrm{w}}=0.7 \mathrm{nT}$, and (c) $B_{\mathrm{w}}=3.0 \mathrm{nT}$. The initial equatorial pitch angle $a_{\text {eq0 }}$ and kinetic energy $E_{k 0}$ of the selected electron are $70^{\circ}$ and $300 \mathrm{keV}$, respectively. $\tau_{\mathrm{b} 0}$ and $\omega_{\mathrm{b} 0}$ are unperturbed period and angular frequency of electron's bounce motion, hereafter. 

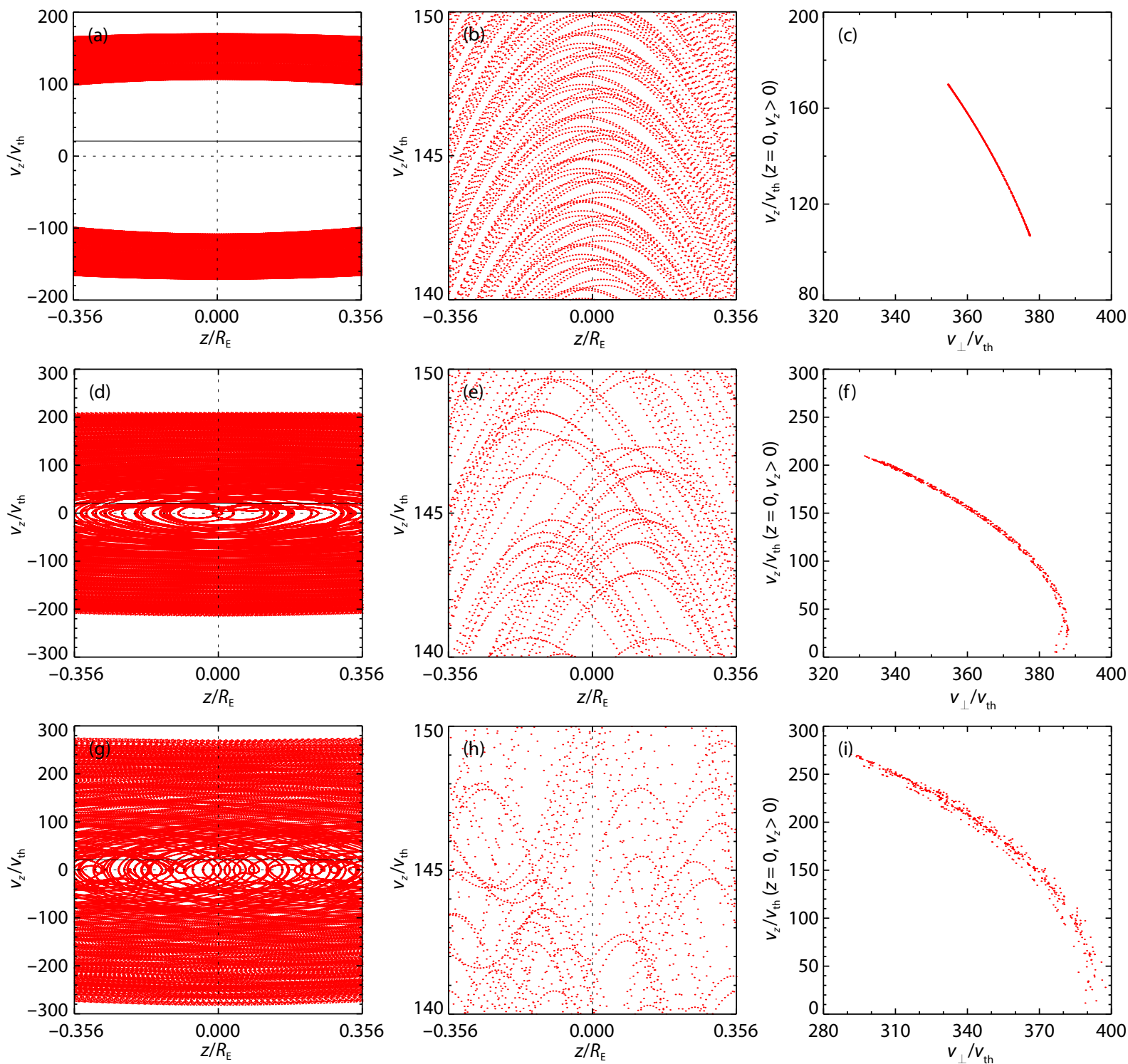

Figure 2. ( $a, b, d, e, g$, and h) The scatterplots of electrons' trajectory in $\left(z, v_{z}\right)$ space. The black line represents Landau resonance velocity as a function of $z$. (c, f, and i) Poincare map of $\left(v_{\perp}, v_{z}\right)$, which is obtained by recording points when the electron crosses the equator from south to north (i.e., $z=0$ and $v_{z}>0$ ). Here the parameters of wave and electron are the same as those in Figure 1.

meters of waves and electrons are the same as those in Figure 1. As expected, when the wave amplitude is small $(0.1 \mathrm{nT})$, the Poincare maps in Figures $2 \mathrm{~b}$ and $2 \mathrm{c}$ only show simple lines, supporting the periodic motion of the electron in this wave field. If the wave amplitude increases to $3 \mathrm{nT}$, then the trajectory of electrons in the Poincare map becomes irregular and the electron motion is chaotic (Figures $2 \mathrm{~h}$ and $2 \mathrm{i}$ ).

Since the bounce resonant scattering effect of MS waves on electrons is sensitive to the initial wave phase (Chen LJ and Bortnik, 2020), the amplitude threshold of electron stochastic motion is also affected by the initial wave phase $\phi_{0}$. Figure 3 a shows the scatterplots of $v_{z}$ by recording points when electrons cross the equator from south to north (i.e., $z=0$, and $v_{z}>0$ ) at different initial wave phase $\phi_{0}$ where the MS wave amplitude is fixed as $B_{\mathrm{w}}=$ $0.4 \mathrm{nT}$, while in $3 \mathrm{~b}$ the amplitude threshold of electron stochastic motion is shown as a function of $\phi_{0}$. The initial pitch angle and en- ergy of electrons are $70^{\circ}$ and $300 \mathrm{keV}$. The horizontal dashed line represents the initial value of $v_{z}$ for electrons at the equator of $L=$ 6.8. The maximum variation in $v_{z}$ caused by the first-order bounce resonance roughly reflects the width of the resonant island. Furthermore, the variation of $v_{z}$ is found to be dependent on the initial wave phase (Figure 3a), which is also equal to the initial phase difference between the electrons and MS waves in our model (Chen LJ and Bortnik, 2020). According to previous studies (Guo $\mathrm{ZH}$ et al., 2008), the stochastic motion of the charged particles can be caused by the overlapping of adjacent resonant islands, suggesting that the larger the resonant islands, the more easily the electron stochastic motion can occur. As shown in Figure $3 \mathrm{~b}$, the amplitude threshold of electron stochastic motion has a minimum of $\sim 0.4 \mathrm{nT}$ at $\phi_{0}=\pi$. Hereafter, the amplitude threshold of electron stochastic motion in the field of one MS wave is given by the minimum value over different initial phases. 

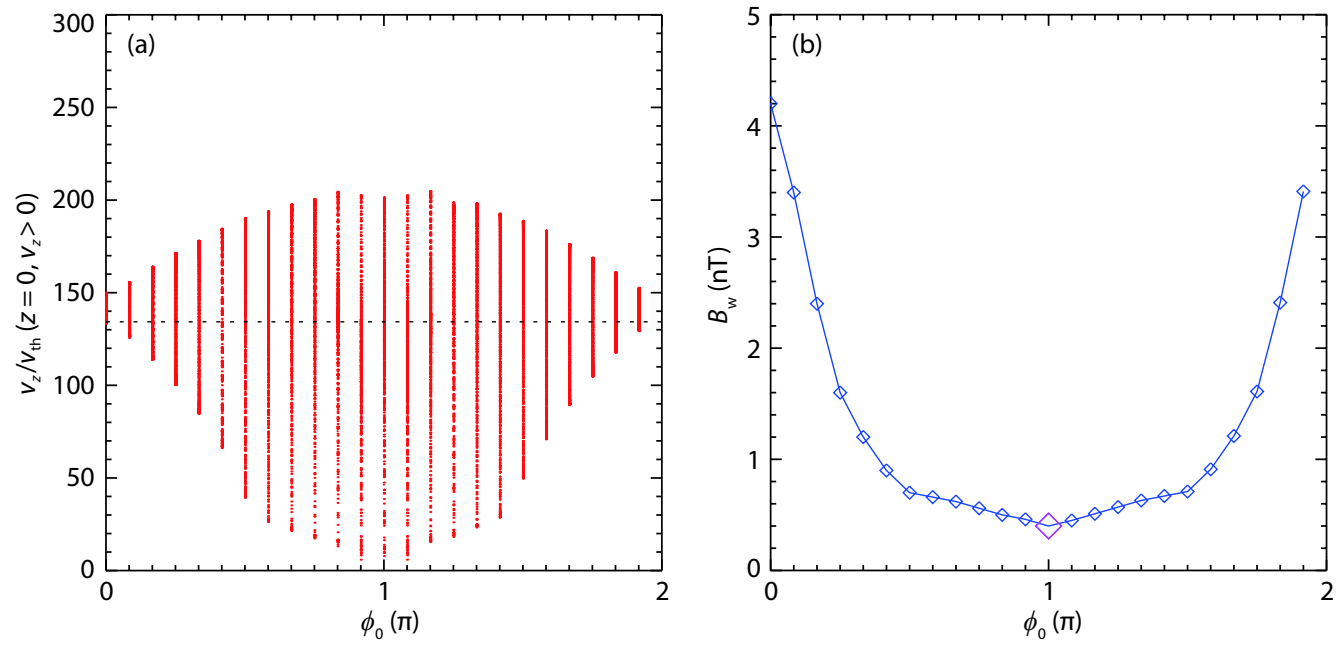

Figure 3. (a) The scatterplots of $v_{z}$ by recording points when the electron crosses the equator from south to north (i.e., $z=0$, and $v_{z}>0$ ) at different initial wave phase $\varphi_{0}$, the amplitude of MS wave is fixed as $B_{\mathrm{w}}=0.4 \mathrm{nT}$, and (b) the amplitude threshold of electron stochastic motion as a function of $\varphi_{0}$. Here the initial pitch angle and electron energy are $70^{\circ}$ and $300 \mathrm{keV}$. The horizontal dashed line represents the initial value of $v_{z}$ for an electron at the equator of $L=6.8$.

The amplitude threshold also depends on the particle parameters; the dependence of the amplitude threshold on the initial pitch angle $a_{\text {eq0 }}$ of the electron is given in Figure 4. Different colors represent the bounce resonance between MS waves and electrons with different orders of $n$. In each simulation run, the frequency of MS waves will be chosen to satisfy the bounce resonance condition $\omega=n \omega_{\mathrm{b}}$, and we fix the initial kinetic energy of electrons as $300 \mathrm{keV}$. The shaded region $\left(83^{\circ} \leq a_{\mathrm{eq} 0} \leq 87^{\circ}\right)$ where the left and right boundaries of $a_{\text {eq0 }}$ are determined by the Landau resonance

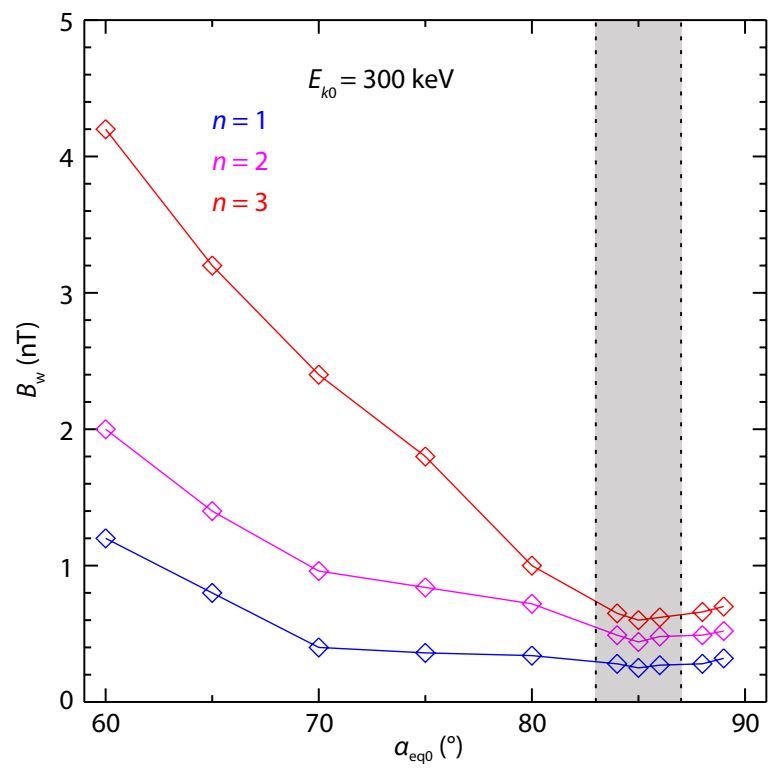

Figure 4. The amplitude threshold of the electron stochastic motion as a function of initial equatorial pitch angle $a_{\text {eq } 0}$ under bounce resonance with different order $n$. The shaded region $\left(83^{\circ} \leq a_{\text {eq } 0} \leq 87^{\circ}\right)$, also named Landau resonance region, is determined by the Landau resonance condition $\omega=k_{/ / / / /}$at $\lambda=3^{\circ}$ and $\lambda=0^{\circ}$. condition $\omega=k_{/ / / / /}$at $\lambda=3^{\circ}$ and $\lambda=0^{\circ}$, is named the Landau resonance region for convenience. If the equatorial pitch angle of the electron falls in this region, then the electrons should experience Landau resonance with the MS waves during their bounce motion. As shown in Figure 4, there is a clear trend that the amplitude threshold of electron stochastic motion is decreasing as the equatorial pitch angle of electrons approaches the Landau resonance region for each bounce resonance order. Interestingly, the amplitude thresholds reach the minimum in the Landau resonance region. These results imply the coexistence of bounce and Landau resonances will facilitate the development of electron stochastic motion. Besides, we find that the amplitude threshold increases proportionally with the bounce resonance order $n$ ( $n=$ 1,2 , and 3). The correlation between bounce resonance order $n$

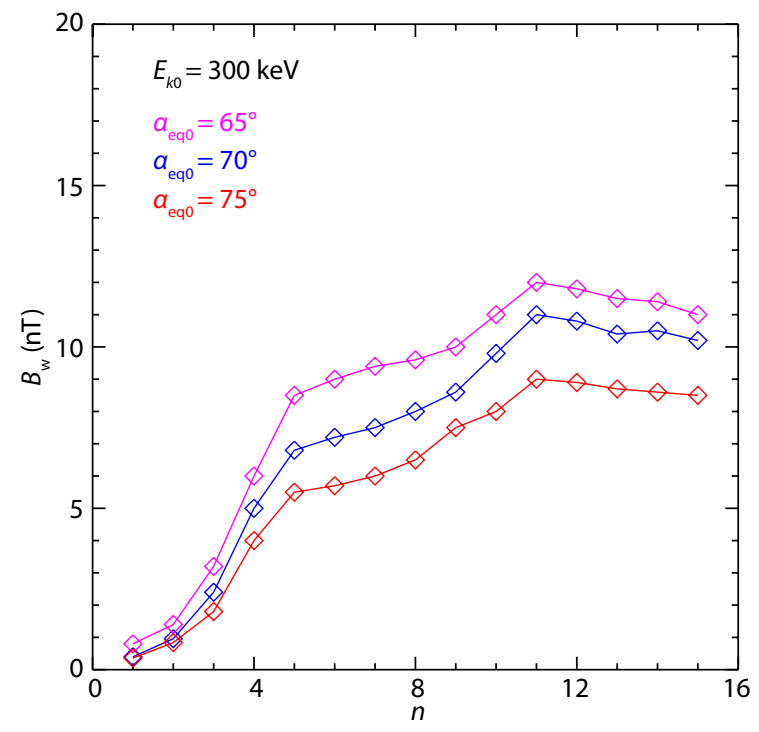

Figure 5. The amplitude threshold of electron stochastic motion at different bounce resonance order $n$ and electron's $a_{\text {eq0 }}$. 
and the amplitude threshold has been thoroughly investigated in Figure 5.

Figure 5 shows the amplitude threshold of electron stochastic motion at different bounce resonance order $n$ and electron $a_{\text {eq0 }}$.
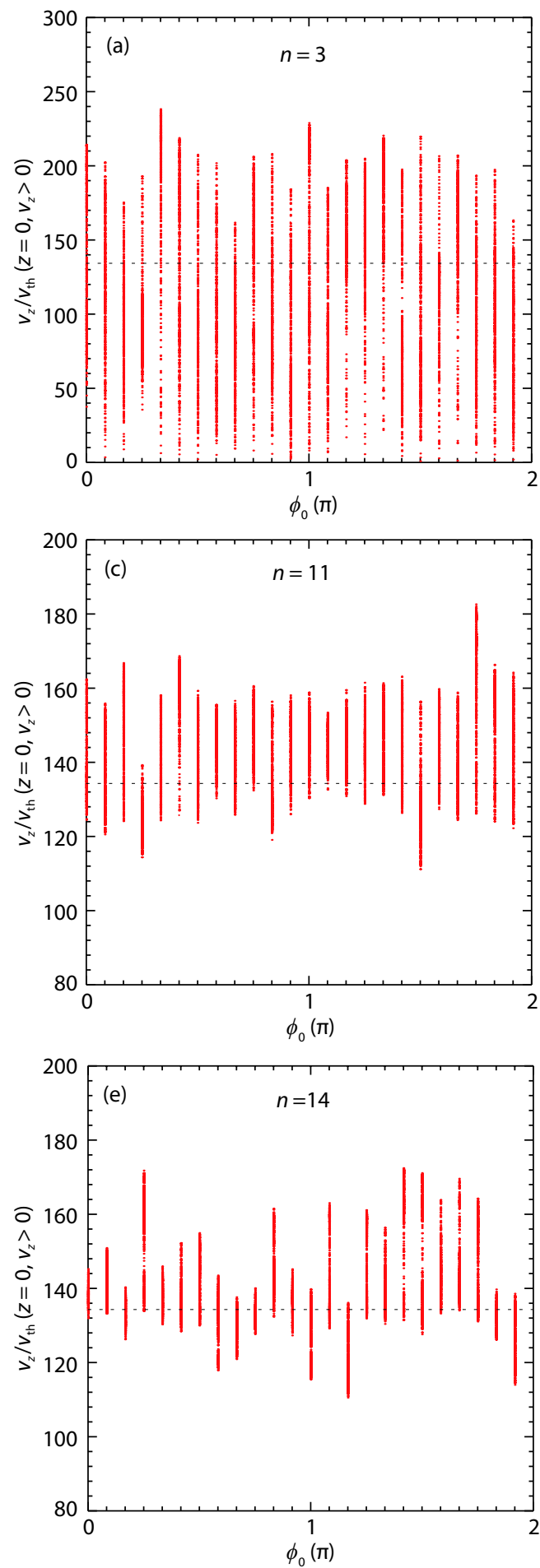

Note that the selected three $a_{\text {eqo }}$ are far away from the Landau resonance region. When MS wave-driven bounce resonance occurs at the low order $(n \leq 11)$, the amplitude threshold tends to become larger with the increase of $n$. However, if the bounce reson-
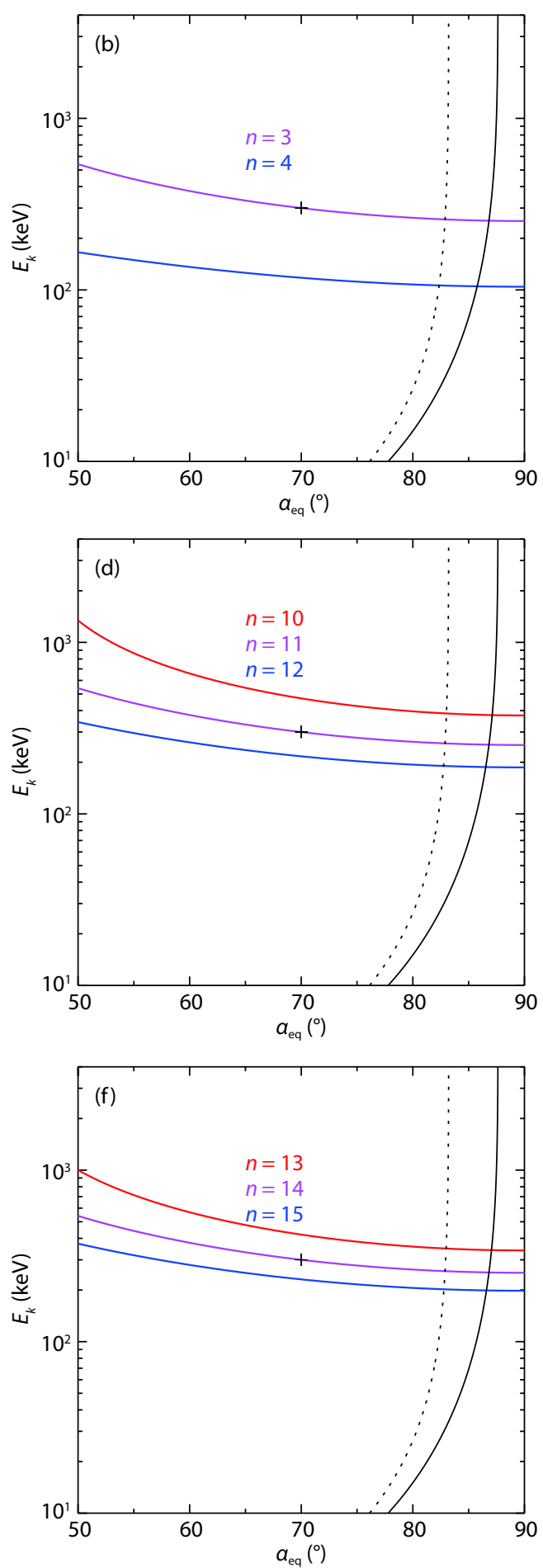

Figure 6. (a, c, and e) The scatterplots of $v_{z}$ in the same format as Figure 3a for bounce resonance order $n=3,11$, and 14, respectively. The wave amplitude is fixed as $B_{\mathrm{w}}=5.0 \mathrm{nT}$. ( $\mathrm{b}, \mathrm{d}$, and $\mathrm{f}$ ) The bounce and Landau resonance conditions in the $\left(a_{\mathrm{eq}} E_{k}\right)$ plane for the monochromatic MS wave with frequency $\omega=3 \omega_{\mathrm{b} 0}, 11 \omega_{\mathrm{b} 0}$, and $14 \omega_{\mathrm{b} 0}$, respectively. The lines of coded color denote the bounce resonance conditions, while the black solid and dotted lines indicate the Landau resonance conditions at $\lambda=0^{\circ}$ and $\lambda=3^{\circ}$, respectively. The black plus marks the initial position of the selected electron, whose $a_{\mathrm{eq} 0}$ and $E_{k 0}$ are $70^{\circ}$ and $300 \mathrm{keV}$. 
ance order $n$ is over 11 , then an increase of $n$ will reduce the amplitude threshold. To better understand the dependence of the stochastic threshold on bounce resonance order, further detail is given in Figure 6 . Figures $6 a, 6 c$, and 6 e show the scatterplot of $v_{z}$ in the same format as Figure 3 a for bounce resonance order $n=3$, 11 , and 14 , respectively. The wave amplitude is fixed as $B_{\mathrm{w}}=$ $5.0 \mathrm{nT}$. Figures $6 \mathrm{~b}, 6 \mathrm{~d}$, and $6 \mathrm{f}$ present the bounce and Landau resonance conditions in the $\left(a_{\mathrm{eq}}, E_{k}\right)$ plane for MS waves with frequency $\omega=3 \omega_{\mathrm{b} 0}, 11 \omega_{\mathrm{b} 0}$, and $14 \omega_{\mathrm{b} 0}$, respectively. Here, $\omega_{\mathrm{b} 0}$ is the unperturbed bounce frequency of the selected electron, whose $a_{\text {eq } 0}$ and $E_{k 0}$ are $70^{\circ}$ and $300 \mathrm{keV}$. In Figure $6 \mathrm{~b}, 6 \mathrm{~d}$, and $6 \mathrm{f}$, the colored lines denote the bounce resonance conditions, while the black solid and dotted lines indicate the Landau resonance conditions at $\lambda=0^{\circ}$ and $\lambda=3^{\circ}$, respectively. The black plus marks the initial position of the selected electron in the $\left(a_{\mathrm{eq}} E_{k}\right)$ plane. FromFigure $6 a$ to $6 c$, we find the maximum $v_{z}$ variation caused by the bounce resonance becomes weaker with the increase of $n$, but remains almost the same level if $n$ is larger than 11 (Figure 6e). This indicates that the width of the bounce resonance island is significantly reduced with increasing resonance order for lower orders (< $11)$, but remains nearly unchanged for higher orders ( $>11)$. From Figure $6 b, 6 d$, to $6 f$, it is clear that the spacing between adjacent bounce resonances in energy is declining with the increase of resonance order, suggesting that the bounce resonance islands are much closer to each other (i.e., the electron energy change caused by the bounce resonance will make the adjacent resonant islands overlap more easily) at higher resonance orders. Since the stochastic motion of electrons should be controlled by the two factors, such as the width of resonance island and the spacing between them, we can speculate that at lower bounce resonance orders, i.e., < 11 for this electron, the decrease in the width of resonance island with the increase of resonance order plays a major role in increasing the amplitude threshold. While at higher orders $>11$, the width of resonance islands remains nearly unchanged, however the adjacent islands become closer as $n$ increases, which may lead to reduced amplitude threshold.

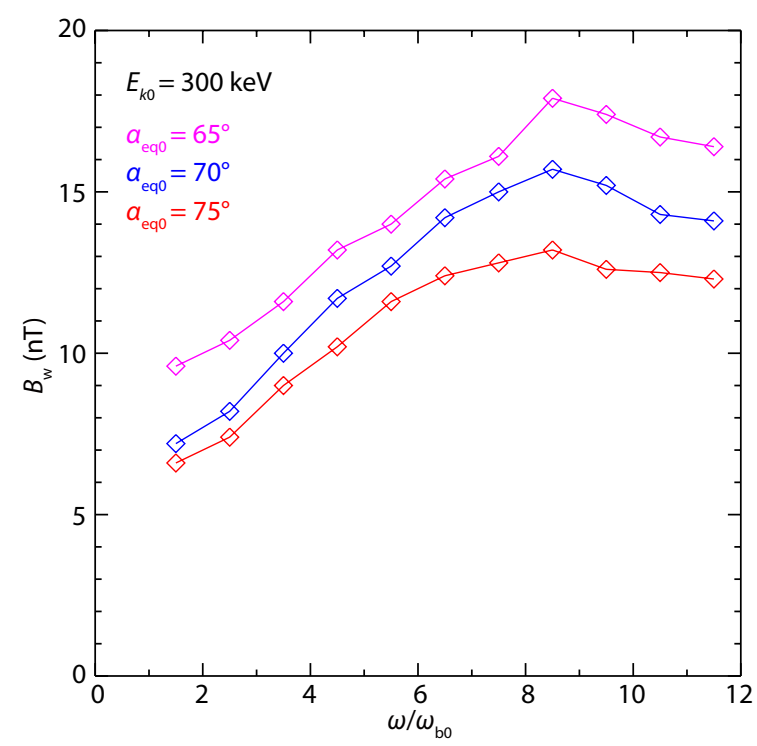

Figure 7. The amplitude threshold as a function of wave frequency for different initial equatorial pitch angles.
To compare with amplitude threshold under resonance, we have also studied the electron stochastic motion when neither the bounce nor Landau resonance condition is satisfied at the beginning. Figure 7 shows the amplitude threshold as a function of wave frequency for different initial equatorial pitch angles; the initial kinetic energy $E_{k 0}$ of the electron is also fixed as $300 \mathrm{keV}$. First off, the amplitude threshold increases with the wave frequency below $8 \omega_{\text {b0 }}$, but gradually decreases above $8 \omega_{\text {b0 }}$, attributed to competition between the width and spacing of resonance islands as discussed above. Moreover, the amplitude thresholds shown in Figure 7 are much larger than those in Figure 5, thus electron stochastic motion is not stimulated easily without the resonant interactions between electrons and MS waves. It is worth noting that, since the high background plasma density condition $\left(\omega_{\mathrm{pe}} / \Omega_{\mathrm{e} 0} \approx 32\right)$ is adopted in our model (Li JX et al., 2014), the transit time effect on electron stochastic motion is quite weak.

\section{Conclusions and Discussions}

Using a test particle simulation method, we studied the stochastic motion of $300 \mathrm{keV}$ electrons in the field of a monochromatic MS wave. If the electrons initially satisfy the bounce resonance with the MS wave, the amplitude threshold of electron stochastic motion initially increases as the bounce resonance order increases, until it peaks at the 11 th order in our study. The amplitude threshold then begins a slow decline. It is remarkable that the coexistence of bounce and Landau resonances between electrons and MS waves will significantly reduce the amplitude threshold. In some cases, the electrons' motion can become stochastic in the field of MS waves with amplitudes below $1 \mathrm{nT}$, suggesting that electron stochastic motion can be caused by intense MS waves in the Earth's magnetosphere, violating the assumption of quasi-linear theory. If neither the bounce nor Landau resonance condition is satisfied initially, the amplitude threshold also exhibits an increasing trend for lower frequencies and a decreasing trend for higher frequencies, however the amplitude threshold is always very large $(>5 \mathrm{nT})$.

Previous studies revealed that the motion of ions in the Alfvén/ion cyclotron or MHD MS wave field can become stochastic when the wave amplitude exceeds a certain threshold, such that the trapping widths associated with neighboring resonances overlap. In this study, we confirm that electron stochastic motion can also be caused by the MS wave, i.e., ion Bernstein mode. Especially with the coexistence of bounce and Landau resonance, electron motion can become stochastic in the field of MS waves with amplitudes below $1 \mathrm{nT}$ in the Earth's magnetosphere. Quasi-linear theory (QLT) is a widely used tool to study the interaction between electron and plasma waves in the Earth's radiation belt; we caution that the validity of QLT may be questionable when intense MS waves are involved.

Previous studies have shown that hundreds of keV electrons can be sufficiently scattered by MS waves via bounce or Landau resonance (Horne et al., 2007; Li JX et al., 2014; Fu S et al., 2019a). Without any preference, we chose $300 \mathrm{keV}$ electrons to study the motion in an MS wave field. For electrons with other energies (e.g., from tens of keV to $1 \mathrm{MeV}$ ), the amplitude threshold of electrons' stochastic motion will change, but their dependences on 
wave and particle parameters are quite similar. Here, for the hundreds of keV electrons with a large pitch angle $\left(60^{\circ}-90^{\circ}\right)$, i.e., with a corresponding bounce period of $\sim 1 \mathrm{~s}$, motion in the low-frequency $(\sim 10 \mathrm{~Hz})$ MS wave field with large amplitude ( several hundreds of pT) may more likely become stochastic in the Earth's magnetosphere. Previous studies showed that the bounce and Landau resonant scattering effects of MS waves on electrons are also sensitive to WNA (Chen LJ et al., 2015; Lei MD et al., 2017). The WNA of MS waves is usually described as a Gaussian distribution with a central peak at $89^{\circ}$ or $89.5^{\circ}$. In this study, the WNA of a monochromatic MS wave is fixed as $89^{\circ}$. If we change the WNA of this wave, the pitch angle range of Landau resonance and amplitude threshold of electron stochastic motion will change, but the amplitude threshold reaches the minimum in the new Landan resonance region. In addition, we focus on equatorial MS waves within $|\lambda| \leq 3^{\circ}$, though off-equatorial MS waves have also been reported with amplitudes up to several hundreds of pT (Ni BB et al., 2018). The electron motion in the strong off-equatorial MS wave field can also become stochastic but may have smaller equatorial pitch angles. For convenience and clearance, we only consider the monochromatic MS wave in our model, but the MS waves in the magnetosphere usually exhibit a discrete or continuous spectrum. The electron stochastic motion in various MS spectra is also interesting and may tend to occur more easily, but that is beyond this paper and requires further investigation.

\section{Acknowledgements}

This research was funded by the Strategic Priority Research Program of Chinese Academy of Sciences Grant No. XDB41000000, the NSFC grant 41774151, 41631071, Key Research Program of Frontier Sciences CAS (QYZDJ-SSW-DQC010), USTC Research Funds of the Double First-Class Initiative (YD3420002001), the Fundamental Research Funds for the Central Universities (WK3420000013), and "USTC Tang Scholar" program. The data used to generate the figures in this paper can be accessed via the following link (http://doi.org/10.5281/zenodo.4133521).

\section{References}

Balikhin, M. A., Shprits, Y. Y., Walker, S. N., Chen, L. J., Cornilleau-Wehrlin, N., Dandouras, I., Santolik O., Carr C., Yearby K. H., and Weiss, B. (2015). Observations of discrete harmonics emerging from equatorial noise. Nat. Commun., 6, 7703. https://doi.org/10.1038/ncomms8703

Boardsen, S. A., Gallagher, D. L., Gurnett, D. A., Peterson, W. K., and Green, J. L. (1992). Funnel-shaped, low-frequency equatorial waves. J. Geophys. Res., 97(A10), 14967-14976. https://doi.org/10.1029/92JA00827

Boardsen, S. A., Hospodarsky, G. B., Kletzing, C. A., Engebretson, M. J., Pfaff, R. F., Wygant, J. R., Kurth W. S., Averkamp T. F., Bounds S. R., ... De Pascuale, S. (2016). Survey of the frequency dependent latitudinal distribution of the fast magnetosonic wave mode from Van Allen Probes Electric and Magnetic Field Instrument and Integrated Science waveform receiver plasma wave analysis. J. Geophys. Res. :Space Phys., 121(4), 2902-2921.

https://doi.org/10.1002/2015JA021844

Boardsen, S. A., Hospodarsky, G. B., Min, K., Averkamp, T. F., Bounds, S. R., Kletzing, C. A., and Pfaff, R. F. (2018). Determining the wave vector direction of equatorial fast magnetosonic waves. Geophys. Res. Lett., 45(16), 7951-7959. https://doi.org/10.1029/2018GL078695

Boris, J. P. (1970). Relativistic plasma simulation-optimization of a hybrid code. In Proceedings of the Fourth Conference on Numerical Simulation on Plasmas (pp. 3-67). Washington, D. C. : Naval Research Laboratory.
Cai, B., Wu, Y. F., and Tao, X. (2020). Effects of nonlinear resonance broadening on interactions between electrons and whistler mode waves. Geophys. Res. Lett., 47(11), e2020GL087991. https://doi.org/10.1029/2020GL087991

Chen, L., Lin, Z. H., and White, R. (2001). On resonant heating below the cyclotron frequency. Phys. Plasmas, 8(11), 4713-4716. https://doi.org/10.1063/1.1406939

Chen, L. J., Thorne, R. M., Jordanova, V. K., and Horne, R. B. (2010). Global simulation of magnetosonic wave instability in the storm time magnetosphere. J. Geophys. Res., 115(A11), A11222. https://doi.org/10.1029/2010JA015707

Chen, L. J., and Thorne, R. M. (2012). Perpendicular propagation of magnetosonic waves. Geophys. Res. Lett., 39(14), L14102. https://doi.org/10.1029/2012GL052485

Chen, L. J., Maldonado, A., Bortnik, J., Thorne, R. M., Li, J. X., Dai, L., and Zhan, X. $Y$. (2015). Nonlinear bounce resonances between magnetosonic waves and equatorially mirroring electrons. J. Geophys. Res. :Space Phys., 120(8), 6514-6527. https://doi.org/10.1029/2015JA021174

Chen, L. J., Sun, J. C., Lu, Q. M., Gao, X. L., Xia, Z. Y., and Zhima, Z. (2016). Generation of magnetosonic waves over a continuous spectrum. J. Geophys. Res. :Space Phys., 121(2), 1137-1147. https://doi.org/10.1002/2015JA022089

Chen, L. J., and Bortnik, J. (2020). Wave-particle interactions with coherent magnetosonic waves. In A. N. Jaynes and M. E. Usanova (Eds. ), Elsevier. https://doi.org/10.1016/B978-0-12-813371-2.00004-4

Fu, S., Ni, B. B., Li, J. X., Zhou, C., Gu, X. D., Huang, S. Y., Zhang, H., Ge, Y. S., and $\mathrm{Cao}, \mathrm{X}$. (2016). Interactions between magnetosonic waves and ring current protons: Gyroaveraged test particle simulations. J. Geophys. Res. :Space Phys., 121(9), 8537-8553. https://doi.org/10.1002/2016JA023117

Fu, S., Ni, B. B., Zhou, R. X., Cao, X., and Gu, X. D. (2019a). Combined scattering of radiation belt electrons caused by Landau and bounce resonant interactions with magnetosonic waves. Geophys. Res. Lett., 46(17-18), 10313-10321. https://doi.org/10.1029/2019GL084438

Fu, S., Ni, B. B., Tao, X., Ge, Y. S., Liu, J., Lou, Y. Q., Gu, X. D., Cao, X., Xiang, Z., ... Zhang, Y. (2019b). Interactions between $\mathrm{H}^{+}$band EMIC waves and radiation belt relativistic electrons: Comparisons of test particle simulations with quasi-linear calculations. Phys. Plasmas, 26(3), 032901. https://doi.org/10.1063/1.5054809

Fu, S., and Ge, Y. S. (2021). Acceleration of ring current protons driven by magnetosonic waves: Comparisons of test particle simulations with quasilinear calculations. Astrophys. J., 908(2), 203. https://doi.org/10.3847/1538-4357/abd2b3

Gao, X. L., Lu, Q. M., Wu, M. Y., and Wang, S. (2012). Ion stochastic heating by obliquely propagating magnetosonic waves. Phys. Plasmas, 19(6), 062111. https://doi.org/10.1063/1.4731707

Gao, X. L., Sun, J. C., Lu, Q. M., Chen, L. J., and Wang, S. (2018). Generation of lower harmonic magnetosonic waves through nonlinear wave-wave interactions. Geophys. Res. Lett., 45(16), 8029-8034. https://doi.org/10.1029/2018GL079090

Gary, S. P., Liu, K. J., and Winske, D. (2011). Bernstein instability driven by suprathermal protons in the ring current. J. Geophys. Res., 116(A8), A08215. https://doi.org/10.1029/2011JA016543

Gates, D. A., Gorelenkov, N. N., and White, R. B. (2001). lon heating by fastparticle-induced Alfven turbulence. Phys. Rev. Lett., 87, 205003. https://doi.org/10.1103/PhysRevLett.87.205003

Guo, Z. H., Crabtree, C., and Chen, L. (2008). Theory of charged particle heating by low-frequency Alfvén waves. Phys. Plasmas, 15(3), 032311. https://doi.org/10.1063/1.2899326

Gurnett, D. A. (1976). Plasma wave interactions with energetic ions near the magnetic equator. J. Geophys. Res., 81(16), 2765-2770. https://doi.org/10.1029/JA081i016p02765

Horne, R. B., Thorne, R. M., Glauert, S. A., Meredith, N. P., Pokhotelov, D., and Santolık, O. (2007). Electron acceleration in the Van Allen radiation belts by fast magnetosonic waves. Geophys. Res. Lett., 34(17), L17107. https://doi.org/10.1029/2007GL030267

Kasahara, Y., Kenmochi, H., and Kimura, I. (1994). Propagation characteristics of the ELF emissions observed by the satellite Akebono in the magnetic equatorial region. Radio Sci., 29(4), 751-767. 
https://doi.org/10.1029/94RS00445

Laakso, H., Junginger, H., Roux, A., Schmidt, R., and de Villedary, C. (1990). Magnetosonic waves above $f_{c}\left(\mathrm{H}^{+}\right)$at geostationary orbit-GEOS 2 results. J. Geophys. Res. :Space Phys., 95(A7), 10609-10621.

https://doi.org/10.1029/JA095iA07p10609

Lei, M. D., Xie, L., Li, J. X., Pu, Z. Y., Fu, S., Ni, B. B., Hua M., Chen L. J., and Li, W. (2017). The radiation belt electron scattering by magnetosonic wave: Dependence on key parameters. J. Geophys. Res. :Space Phys., 122(12), 12338-12352. https://doi.org/10.1002/2016JA023801

Li, J. X., Ni, B. B., Xie, L., Pu, Z. Y., Bortnik, J., Thorne, R. M., Chen, L. J., Ma, Q. L., Fu, S. Y., ... Guo, R. L. (2014). Interactions between magnetosonic waves and radiation belt electrons: Comparisons of quasi-linear calculations with test particle simulations. Geophys. Res. Lett., 41(14), 4828-4834. https://doi.org/10.1002/2014GL060461

Li, J. X., Ni, B. B., Ma, Q. L., Xie, L., Pu, Z. Y., Fu, S. Y., Thorne, R. M., Bortnik, J., Chen, L. J., ... Summers, D. (2016). Formation of energetic electron butterfly distributions by magnetosonic waves via Landau resonance. Geophys. Res. Lett., 43(7), 3009-3016. https://doi.org/10.1002/2016GL067853

Li, X. X., Tao, X., Lu, Q. M., and Dai, L. (2015). Bounce resonance diffusion coefficients for spatially confined waves. Geophys. Res. Lett., 42(22), 9591-9599. https://doi.org/10.1002/2015GL066324

Lichtenberg, A. J. , and Lieberman, M. A. (1992). Regular and Chaotic Dynamics (2nd ed, pp. 87-93). New York: Springer-Verlag. https://doi.org/10.1007/9781-4757-2184-3

Lu, Q. M., and Chen, L. (2009). Ion heating by a spectrum of obliquely propagating low-frequency Alfvén waves. Astrophys. J., 704(1), 743-749. https://doi.org/10.1088/0004-637X/704/1/743

Lyons, L. R., and Williams, D. J. (1984). Quantitative Aspects of Magnetospheric Physics. Dordrecht, Netherlands, Springer. https://doi.org/10.1007/978-94017-2819-5

Ma, Q. L., Li, W., Thorne, R. M., and Angelopoulos, V. (2013). Global distribution of equatorial magnetosonic waves observed by THEMIS. Geophys. Res. Lett., 40(10), 1895-1901. https://doi.org/10.1002/grl.50434

Ma, Q. L., Li, W., Chen, L. J., Thorne, R. M., and Angelopoulos, V. (2014). Magnetosonic wave excitation by ion ring distributions in the Earth's inner magnetosphere. J. Geophys. Res. :Space Phys., 119(2), 844-852. https://doi.org/10.1002/2013JA019591

Ma, Q. L., Li, W., Thorne, R. M., Bortnik, J., Kletzing, C. A., Kurth, W. S., and Hospodarsky, G. B. (2016). Electron scattering by magnetosonic waves in the inner magnetosphere. J. Geophys. Res. :Space Phys., 121(1), 274-285. https://doi.org/10.1002/2015JA021992

Maldonado, A. A., Chen, L. J., Claudepierre, S. G., Bortnik, J., Thorne, R. M., and Spence, H. (2016). Electron butterfly distribution modulation by magnetosonic waves. Geophys. Res. Lett., 43(7), 3051-3059. https://doi.org/10.1002/2016GL068161

Maldonado, A. A., and Chen, L. J. (2018). On the diffusion rates of electron bounce resonant scattering by magnetosonic waves. Geophys. Res. Lett., 45(8), 3328-3337. https://doi.org/10.1002/2017GL076560

Meredith, N. P., Horne, R. B., and Anderson, R. R. (2008). Survey of magnetosonic waves and proton ring distributions in the Earth's inner magnetosphere. $J$. Geophys. Res., 113(A6), A06213. https://doi.org/10.1029/2007JA012975

Min, K., Němec, F., Liu, K. J., Denton, R. E., and Boardsen, S. A. (2019). Equatorial propagation of the magnetosonic mode across the plasmapause: 2-D PIC simulations. J. Geophys. Res. :Space Phys., 124(6), 4424-4444. https://doi.org/10.1029/2019JA026567

Ni, B. B., Zou, Z. Y., Fu, S., Cao, X., Gu, X. D., and Xiang, Z. (2018). Resonant scattering of radiation belt electrons by off-equatorial magnetosonic waves. Geophys. Res. Lett., 45(3), 1228-1236. https://doi.org/10.1002/2017GL075788

Perraut, S., Roux, A., Robert, P., Gendrin, R., Sauvaud, J. A., Bosqued, J. M., Kremser, G., and Korth, A. (1982). A systematic study of ULF waves above $F_{H+}$ from GEOS 1 and 2 measurements and their relationships with proton ring distributions. J. Geophys. Res., 87(A8), 6219-6236. https://doi.org/10.1029/JA087iA08p06219

Russell, C. T., Holzer, R. E., and Smith, E. J. (1970). OGO 3 observations of ELF noise in the magnetosphere 2 . The nature of the equatorial noise. $J$.
Geophys. Res., 75(4), 755-768. https://doi.org/10.1029/JA075i004p00755

Santolík, O., Pickett, J. S., Gurnett, D. A., Maksimovic, M., and Cornilleau-Wehrlin, N. (2002). Spatiotemporal variability and propagation of equatorial noise observed by Cluster. J. Geophys. Res., 107(A12), 1495. https://doi.org/10.1029/2001JA009159

Santolík, O., Němec, F., Gereová, K., Macúšová, E., de Conchy, Y., and CornilleauWehrlin, N. (2004). Systematic analysis of equatorial noise below the lower hybrid frequency. Ann. Geophys., 22, 2587-2595. https://doi.org/10.5194/angeo-22-2587-2004

Schulz, M., and Lanzerotti, L. J. (1974). Particle Diffusion in the Radiation Belts. Berlin, Heidelberg, Springer. https://doi.org/10.1007/978-3-642-65675-0

Shprits, Y. Y. (2009). Potential waves for pitch-angle scattering of nearequatorially mirroring energetic electrons due to the violation of the second adiabatic invariant. Geophys. Res. Lett., 36(12), L12106. https://doi.org/10.1029/2009GL038322

Smith, G. R., and Kaufman, A. N. (1978). Stochastic acceleration by an obliquely propagating wave-An example of overlapping resonances. Phys. Fluids, 21(12), 2230-2241. https://doi.org/10.1063/1.862161

Sun, J. C., Gao, X. L., Chen, L. J., Lu, Q. M., Tao, X., and Wang, S. (2016a). A parametric study for the generation of ion Bernstein modes from a discrete spectrum to a continuous one in the inner magnetosphere. I. Linear theory. Phys. Plasmas, 23(2), 022901. https://doi.org/10.1063/1.4941283

Sun, J. C., Gao, X. L., Lu, Q. M., Chen, L. J., Tao, X., and Wang, S. (2016b). A parametric study for the generation of ion Bernstein modes from a discrete spectrum to a continuous one in the inner magnetosphere. II. Particle-incell simulations. Phys. Plasmas, 23(2), 022902. https://doi.org/10.1063/1.4941284

Sun, J. C., Chen, L. J., Wang, X. Y., Boardsen, S., Lin, Y., and Xia, Z. Y. (2020). Particle-in-cell simulation of rising-tone magnetosonic waves. Geophys. Res. Lett., 47(18), e2020GL089671. https://doi.org/10.1029/2020GL089671

Tao, X., Bortnik, J., Albert, J. M., and Thorne, R. M. (2012). Comparison of bounceaveraged quasi-linear diffusion coefficients for parallel propagating whistler mode waves with test particle simulations. J. Geophys. Res., 117(A10), A10205. https://doi.org/10.1029/2012JA017931

Tao, X., and Li, X. (2016). Theoretical bounce resonance diffusion coefficient for waves generated near the equatorial plane. Geophys. Res. Lett., 43(14), 7389-7397. https://doi.org/10.1002/2016GL070139

Teng, S. C., Li, W., Tao, X., Ma, Q. L., and Shen, X. C. (2019). Characteristics and generation of low-frequency magnetosonic waves below the proton gyrofrequency. Geophys. Res. Lett., 46(21), 11652-11660. https://doi.org/10.1029/2019GL085372

Thorne, R. M. (2010). Radiation belt dynamics: The importance of wave-particle interactions. Geophys. Res. Lett., 37(22), L22107. https://doi.org/10.1029/2010GL044990

Tsurutani, B. T., Falkowski, B. J., Pickett, J. S., Verkhoglyadova, O. P., Santolik, O., and Lakhina, G. S. (2014). Extremely intense ELF magnetosonic waves: A survey of polar observations. J. Geophys. Res. :Space Phys., 119(2), 964-977. https://doi.org/10.1002/2013JA019284

Villalón, E., and Burke, W. J. (1987). Relativistic particle acceleration by obliquely propagating electromagnetic fields. Phys. Fluids, 30(12), 3695. https://doi.org/10.1063/1.866406

White, R., Chen, L., and Lin, Z. H. (2002). Resonant plasma heating below the cyclotron frequency. Phys. Plasmas, 9(5), 1890-1897. https://doi.org/10.1063/1.1445180

Xiao, F. L., Yang, C., Su, Z. P., Zhou, Q. H., He, Z. G., He, Y. H., Baker, D. N., Spence, H. E., Funsten, H. O., and Blake, J. B. (2015). Wave-driven butterfly distribution of Van Allen belt relativistic electrons. Nat. Commun., 6(1), 8590. https://doi.org/10.1038/ncomms9590

Yu, J., Li, L. Y., Cui, J., Cao, J. B., and Wang, J. (2019a). Effect of low-harmonic magnetosonic waves on the radiation belt electrons inside the plasmasphere. J. Geophys. Res. :Space Phys., 124(5), 3390-3401. https://doi.org/10.1029/2018JA026328

$\mathrm{Yu}$, J., Wang, J., and Cui, J. (2019b). Ring current proton scattering by lowfrequency magnetosonic waves. Earth Planet. Phys., 3(4), 365-372. https://doi.org/10.26464/epp2019037 